\title{
Comparative Analysis of Kinematics of the Last Exertion Technique of Chinese Elite Female Discus Throwers
}

\author{
Jianhong Xu \\ Chengdu Sport Institute \\ Chengdu, China \\ 903409781@qq.com
}

\author{
Weiwei Zhang \\ Chengdu Sport Institute \\ Chengdu, China \\ 1029310304@qq.com
}

\begin{abstract}
The article uses the literature data method to review and collate relevant literature materials at home and abroad, and through on-the-spot shooting of the discus throwing skills of the women discus athletes Feng Bin, Su Xinyue and Chen Yang in the April 2018 national track and field throwing series. This paper uses three-dimensional video analysis software to perform a point-analysis on the last forceful technical movement of their best performance in the game, and obtains a comparative analysis of relevant kinematic parameters. The study concludes that: first, the initial speed of the three discus throwers is slow, and there is a certain gap between them and the world's top athletes; Feng Bin and Su Xinyue's shot angle is more reasonable than Chen Yang; second, in the final exertion phase, Feng Bin and Su Xinyue spent less time than Chen Yang and completed the action process faster. At the time of the left foot landing, Feng Bin and Su Xinyue had better shoulder and hip angle parameters than Chen Yang. Chen Yang did not form a good surpass. The posture of the device, discus throwing moment, Su Xinyue's pull lead angle is higher than Feng Bin and Chen Yang, her technology is more reasonable. In the final stage of exertion, the two-legged station is far from Feng Binyao than Su Xinyue and Chen Yang, leaving enough room for the body to move.
\end{abstract}

Keywords-Women's discus; Final force technique; Comparative analysis of kinematics

\section{RESEARCH PURPOSES}

This article analyzes the kinematics of the final exertion techniques of Chinese women discus throwers Feng Bin, Su Xinyue, and Chen Yang in the April 2018 national track and field throwing series to find out their strengths and weaknesses in the final exertion technique at this stage. Then, based on the actual situation, compare the relevant parameters with those of domestic and foreign elite athletes, and propose corresponding countermeasures to provide a theoretical basis for mobilizing training plans in future training and further improve the performance of sports.

\section{RESEARCH OBJECTS AND METHODS}

\section{A. Research object}

Feng Bin, whose height is $178 \mathrm{~cm}$, is an athlete of the

People's Liberation Army and an athlete whose rank is international. The results of this analysis is 62.11 meters.

$\mathrm{Su}$ Xinyue, whose height is $178 \mathrm{~cm}$, is an athlete of Hebei Anda and an athlete whose rank is international. The result of this analysis is 61.87 meters.

Chen Yang, whose height is $180 \mathrm{~cm}$, is an athlete of Hebei Anda and an international athlete. The result of this analysis is 59.60 meters.

\section{B. Research methods}

Using two JVC GC-PX10AC HD cameras (50 frames per second) to take pictures of Feng Bin, Su Xinyue, and Chen Yang's technical moves in the National Open Draw Discus Throwing Competition held in April 2018 in Xipu, Chengdu, China. The No. 1 machine is placed on the left side of the throwing circle, the No. 2 machine is directly behind the throwing circle. The angle between the main optical axes of the two cameras is about $90^{\circ}$. Using the Star High Ti 3-D Signal TEC V3.2HDC video analysis software, the best score for them is. Three-dimensional analysis of technical force at the end of force technology. Low-pass digital filtering was used to smooth the raw data, the cut-off frequency was $8 \mathrm{~Hz}$, and the obtained kinematics parameters were compared and analyzed.

\section{RESEARCH RESULTS AND ANALYSIS}

\section{A. Distinguishing the stage of discus throwing technique}

In order to better describe and analyze the technical movements of the athletes, the last force technique is divided into two phases: one stage and the other.

Time division: T1: Left foot landing moment T2: Discus shot moment

Stage division: T1-T2: final exertion stage 


\section{B. Discus throwing technique phase parameter interpretation}

Shoulder and hip angle: The angle between the horizontal axis of the shoulder and the transverse axis of the hip.

Hip angle: The angle formed between the ipsilateral shoulder joint and the knee joint at the hip joint.

Lead angle: The angle between the line from the left shoulder to the right shoulder and the line from the right shoulder to the center of the instrument.
Shooting angle: At the moment of discus throwing, the angle between the discus speed direction and the horizontal plane.

Shooting height: At the moment of discus throwing, the vertical height of the midpoint of the wrist and the ground.

C. The contrast and analysis of velocity and angle parameters of discus throwing at the last hardening stage

TABLE I.

Comparative ANAlysis of SPEEd AND ANGLe Parameters of Athletes' SHOt in Discus ShoOting

\begin{tabular}{cccc}
\hline Names & Feng Bin & Su Xinyue & Chen Yang \\
\hline Initial speed $(\mathrm{m} / \mathrm{s})$ & 24.10 & 23.21 & 23.09 \\
Shooting angle $\left({ }^{\circ}\right)$ & 38.3 & 39.1 & 42.6 \\
\hline
\end{tabular}

The final stage of technical application has a crucial influence on the performance of the discus project. Whether the athlete can exert the fastest shot speed and get the best shot angle and height directly depends on the technical exertion of the final exertion phase [1]. The literature shows that the initial speed of the device is the most important factor among the three variables of the shot angle, the height of the device's centroid shot and the initial speed of the shot. Research shows that, under certain conditions, the initial speed of the shot is increased by $1 \mathrm{~m} / \mathrm{s}$. The score will increase by $4.8 \mathrm{~m}$.

According to the values of Table 1, at the time of (T2), the initial speed of the three athletes was relatively close, of which Feng Bin's initial speed was the highest, reaching $24.10 \mathrm{~m} / \mathrm{s}$. The initial speed of Su Xinyue and Chen Yang was $0.89 \mathrm{~m} / \mathrm{s}$ slower than Feng Bin's initial speed. $\mathrm{s}$ and $1.01 \mathrm{~m} / \mathrm{s}$, which is the main reason why Feng Bin's performance is better than the

other two athletes. The average speed of the world's best women discus throwers is $25.0 \mathrm{~m} / \mathrm{s}$, which is a big difference compared to that of the world. In addition, the shot angle is also one of the main factors affecting discus performance. At present, some scholars believe that the best shot angle for female discus is $35^{\circ}-40^{\circ}$. It can be seen from Table 1 that the three athletes' shot angles are $38.3^{\circ}, 39.1^{\circ}$, and $42.6^{\circ}$, respectively. Only Feng Bin and Su Xinyue's shot angles are within the best shot angle range. The best shot angle for the world's top female discus athletes is around $35^{\circ}$ (Blowa $\left.67.40 \mathrm{~m}, 34.6^{\circ}\right)$.

\section{Comparison Analysis of Athletes' Shooting Height and Time of Final Exertion}

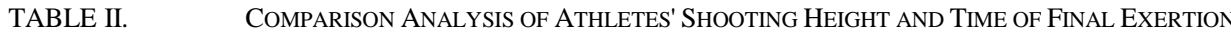

\begin{tabular}{cccc}
\hline Names & Feng Bin & Su Xinyue & Chen Yang \\
\hline Stage time $(s)$ & 0.18 & 0.20 & 0.22 \\
Shooting height $(m)$ & 1.96 & 1.57 & 1.61
\end{tabular}

The shooting speed and angle are certain. The higher the shooting height is, the longer the flying time is, and the greater the flight distance and maximum height of the instrument are [2].

According to the values of Table 2, final exertion phase, Feng Bin spent $0.18 \mathrm{~s}$ in total, completing the movement process faster than the other two athletes. The research data shows that the current reasonable time interval for the world's best women discus throwers is between $0.10 \mathrm{~s}$ and $0.20 \mathrm{~s}$.

Compared with other variables, the athlete's shot height has the least impact on the throwing performance during the entire throwing process because it has a great relationship with the height of the player. From Table 2, we can see that Feng Bin's shot height is $1.96 \mathrm{~m}$, which is far more than the other two players. She is tall because her own height reaches $1.84 \mathrm{~m}$.

E. Analysis of Parameters of Athlete's Shoulder, Shoulder, And Pulling Angle in the Last Force Stage

TABLE III.

Comparative ANALysis of Parameters of Athlete's Shoulder HiP ANGLE AND PUlling ANGLe

\begin{tabular}{lllll}
\hline Names & & Feng Bin & Su Xinyue & ChenYang \\
\hline \multirow{2}{*}{ Shoulder and hip angle $\left(^{\circ}\right)$} & $\mathrm{T} 1$ & 50.7 & 47.2 & 42.4 \\
& $\mathrm{~T} 2$ & 12.5 & 15.7 & 18.1 \\
\cline { 2 - 5 } Lead angle $\left({ }^{\circ}\right)$ & $\mathrm{T} 1$ & 147.8 & 141.3 & 143.6 \\
& $\mathrm{~T} 2$ & 170.3 & 178.7 & 165.5
\end{tabular}

From the moment of the landing of the left foot to the moment of discus throwing, the shoulder angle of the athlete gradually decreases, and the magnitude of the decrease

indicates the magnitude of the body rotation of the athlete during the final exertion phase. At the moment of discus throw athletes pull The size of the horn is positively correlated with 
the horizontal distance of the discus flight, so in order to obtain excellent athletic performance, sports. The pulling angle should be close to $180^{\circ}$ [3].

According to the values of Table 3, Feng Bin and $\mathrm{Su}$ Xinyue are on the left foot (T1), and the shoulder angle is $50.7^{\circ}$.Compared with $47.2^{\circ}$, there is not much difference between the two, but Chen Yang is $42.4^{\circ}$. There is a certain gap between the two, indicating that Chen Yang did not complete the movement of the hip shaft beyond the shoulder axis at the time of the landing of the left foot and did not form a good one. Ultra-distant instrument posture. At the same time, the difference between the left foot landing time (T1) and discus throwing time (T2) shoulder angles of the three athletes was $38.2^{\circ}, 31.5^{\circ}$, and $24.3^{\circ}$, respectively. Among them, Feng Bin's difference was the largest, indicating her final exertion phase rotation. Larger, better use of torso rotation added discus last shot speed. In addition, at the time of (T2), Feng Bin's lead angle is $170.3^{\circ}$, Su Xinyue is $178.7^{\circ}$, Chen Yang is $165.5^{\circ}$, Feng Bin and Su Xinyue's lead angle are closer to $180^{\circ}$, and Chen Yang's pull is cited. The angle is the smallest, indicating her early appearance.

\section{F. Analysis of the distance between the two feet when the athlete left the ground when the final effort is applied}

TABLE IV. ANALYSIS OF THE DISTANCE BETWEEN THE ATHLETES' FEET WHEN THE LEFT FOOT IS ON THE GROUND

\begin{tabular}{llll}
\hline Names & Feng Bin & Su Xinyue & Chen Yang \\
\hline
\end{tabular}

Distance between feet $(m)$

0.71

Finally, the two-foot position of the exertion phase can indirectly assess the quality of the athlete's technique. The correct position and reasonable distance between the two legs are conducive to the athlete's final twist and force [4]. When throwing discus, it is required that the distance between the two feet should be appropriate, which is conducive to the forcefully accelerated distance in the horizontal direction. When the legs are too narrow, it is easy to cause the last stage of the throwing force to move upwards and forwards; the legs are too wide to open, which makes it difficult to support the force of the two legs and the movement of the center of gravity of the body [5].

According to the values of Table 4,left foot landing time (T1) Feng Bin's two-foot distance is $0.71 \mathrm{~m}$, because she is taller than her two athletes, so she is the third among the athletes. This facilitates the muscular contraction of the muscles and completes the whipping action in the final exertion phase to achieve better athletic performance. The intervals between $\mathrm{Su}$ Xinyue and Chen Yang are $0.65 \mathrm{~m}$ and $0.61 \mathrm{~m}$ respectively (an average of $0.67 \mathrm{~m}$ for domestic elite athletes). Therefore, from the data, Feng Bin's position on the left foot is more reasonable.

\section{CONCLUSIONS AND SUGGESTIONS}

From early to speed, three players out initial speed 24.1 $\mathrm{m} / \mathrm{s}, 23.21 \mathrm{~m} / \mathrm{s}, 23.09 \mathrm{~m} / \mathrm{s}$, and the excellent female discus athletes in the world to initial speed and a large gap compared to $25.0 \mathrm{~m} / \mathrm{s}$. From the Angle of shot, three players only Feng Bin and $\mathrm{Su}$ Xinyue Angle at the best Angle range (women's discus the best Angle of $35^{\circ}, 40^{\circ}$ ), while chinyo Angle is slightly big, is also one of the cause of her throwing bad grades. Therefore, in the future training, the three athletes should strengthen the special explosive training of hand action, ensure the continuity of technical action, and improve the speed of the discus. Maintain good transcendental movement and keep the throwing arm behind the body as much as possible. During the training, the main goal is to increase the initial speed of shooting, strengthen the training of special explosive force of the shooting action, appropriately increase the distance of accelerated operation of the equipment, and
0.65

0.61

improve the effective transitional connection and consistent speed of the engagement phase and the final exertion phase.

From the point of the last stage when, Feng Bin and the whole process took the shortest $\mathrm{Su}$ Xinyue, $0.18 \mathrm{~s}$ and $0.20 \mathrm{~s}$, respectively, and the world outstanding women's discus athletes phase takes $0.10 \mathrm{~s}$ to $0.10 \mathrm{~s}$, a sign that they better to complete the process, as far as possible to reduce the loss of speed. Chen Yang's phase took 0.22s, which took a little longer. He failed to effectively apply the speed of the transition phase to the final shot phase. From the height of the shot, feng bin because of height, so high shot height, su xinyue and Chen Yang hand height is relatively close. In the final stage of the hard discus running time, poor acceleration will affect the final shot of the whipping action, resulting in an unsatisfactory speed of the results. It is necessary to strengthen the control ability of discus throwing and increase the distance of discus running in the final exertion phase to improve the acceleration ability of discus. Therefore, in future training, Chen Yang should pay attention to better handling the transition stage and the final exertion stage, and shorten the final exertion time. To further improve the order of reasonable force of discus athletes, and to accelerate and brake the various parts of the body from bottom to top, forming the best posture before the discus shot, to ensure that the speed and momentum can be transmitted to the discus.

From the point of the shoulder and hip Angle of athletes left foot touchdown moment, three players is $50.7^{\circ}$ and $47.2^{\circ}$ and $42.4^{\circ}$ respectively, because of his left foot touchdown moment shoulder axis and hip axis horizontal numerical description pulp ahead of the shoulder, the more the greater the Angle from the data, Feng Bin and Su Xinyue than chinyo better in this respect, better formed beyond the action of instrument, fully prepared for the final stage; Chen Yang's final efforts to prepare for action, the body tightened and exceeded the level of discus is not enough, discus throwing technology is not reasonable, landing more hasty or the body center of gravity is too early to move in the direction of the throw. From discus to moments of lead Angle, three players $170.3^{\circ}$ and $178.7^{\circ}$ and 170.3 , respectively, because the last discus to moment, in order to ensure full stretch body, Angle 
of pull the best Angle is close to or exceed $180^{\circ}$, so there is no shoulder, hip, arm chinyo reserves by the elastic potential energy released in full. Therefore, in the future training, athletes should do some specific flexibility training, to practice the movement of surpassing equipment, in order to improve the performance of sports.

At the end of the exertion phase, the two athletes' feet spacing was $0.71 \mathrm{~m}, 0.65 \mathrm{~m}$, and $0.61 \mathrm{~m}$ respectively (an average of $0.67 \mathrm{~m}$ for domestic elite athletes), because Feng Bin's height was higher, so she stood on two feet. The spacing is relatively large, leaving enough room for the body to move. The distance between Su Xinyue and domestic elite athletes is relatively close; Chen Yang and the two have a certain gap, Too close distance between the two legs is not conducive to the muscular contraction, and ultimately affect the final force of the whipping action. In future training, athletes should increase the distance between the two feet, increase the support area, and better maintain the balance of body and mind.

\section{REFERENCES}

[1] QinJianjie,Li Jidong.Kinematic analysis of $\mathrm{Su}$ Xinyue's throwing technique for Chinese women discus throwers[J]. Journal of Shandong Physical Education Institute,2016,32(02):84-89.

[2] Wen Yang,Du Liping.Study on Technology and Mechanical Properties of Discus Throwing Project[J].Journal of Wuhan Institute of Physical Education, 2003(03):51-52+58.

[3] Li Wenze. Kinematic analysis of Su Xinyue's throwing technique of Chinese female discus thrower [D]. Chengdu Institute of Physical Education, 2016.

[4] Zhang Chunlian,Jin Xiaoning,Cao Qi.Discussion on the Integrality of Discus Throwing Technique[J]. Track and Field,2002(07):29.

[5] Song Yunfei. Contrast and analysis of the final exertion of Chinese female discus throwers [D]. Shandong Normal University, 2013. 